REFERENCES:

[1] van Vollenhoven, et al. Lancet. 2018:392:1330.

[2] Touma Z, et al. EULAR June 2018, Amsterdam, NL.

[3] Touma Z, et al. APLAR. April 2019, Brisbane, Australia.

Disclosure of Interests: Zahi Touma Grant/research support from: GSK Canada, Consultant for: UBC, Pfizer, Janssen, Inc, Dafna D Gladman Grant/research support from: AbbVie, Amgen, Celgene, Lilly, Novartis, Pfizer, and UCB, Consultant for: AbbVie, Amgen, BMS, Celgene, Galapagos, Gilead, Janssen, Lilly, Novartis, Pfizer, and UCB, Shawn Rose Employee of: Janssen Research \& Development, LLC, Kaiyin Fei Employee of: Janssen Research \& Development, LLC, Y Irene Gregan Employee of: Janssen Research \& Development, LLC, Robert Gordon Employee of: Janssen Research \& Development, LLC, Kim Hung Lo Employee of: Janssen Research \& Development, LLC, Murray B Urowitz Grant/research support from: GSK, Consultant for: BMS, Celgene, GSK, Lilly, UCB

DOI: 10.1136/annrheumdis-2019-eular.230

\section{THU0251 ASSOCIATION OF SMOKING STATUS AND TOTAL AND INDIVIDUAL DAMAGE INDEX IN SYSTEMIC LUPUS ERYTHEMATOSUS}

Romy Kallas, LI Jessica, Michelle A Petri. Johns Hopkins University, School of Medicine, Baltimore, United States of America

Background: Smoking is a risk factor for systemic lupus erythematosus (SLE). It has been associated with increased disease activity and decreased effectiveness of hydroxychloroquine in cutaneous lupus.

Objectives: The objective of the study was to determine the association between smoking status and total and individual damage items in SLE.

Methods: We analyzed data from the Hopkins Lupus Cohort. Damage was recorded using the Systemic Lupus Erythematosus International Collaborating Clinics/American College of Rheumatology (SLICC/ACR) Damage Index. Fisher's exact test and Wilcoxon test were used in exploratory analysis. Logistic regression was used to estimate the association between damage and smoking status (ever/never). Odds ratios and $95 \%$ confidence intervals were reported. Stratification by ethnicity was done for individual damage items that were found to be significantly associated with smoking.

Results: The prevalence of ever smokers in our cohort was $36 \%$. SLE patients who ever smoked had higher odds of total damage with higher mean total damage index scores $(p<0.0001)$. Data for individual damage items significantly associated with smoking are presented in Table 1.

The association between cataract and smoking was still present after adjusting for ethnicity, diabetes, and prednisone use in a multivariate regression model $(\mathrm{OR}=1.4, \mathrm{p}=0.0083$ ).

Stratification by ethnicity showed that African- American SLE patients who ever smoked were more likely to have cataract, scarring chronic alopecia, extensive scarring, skin ulceration, pulmonary hypertension, cerebrovascular events, cardiomyopathy, and diabetes compared to non-smokers. Caucasian SLE patients who ever smoked were more likely to have extensive scarring, gastrointestinal infarction and resection, and muscle atrophy (Table 1)

Abstract THU0251 - Table 1. Relationship between SLICC/ACR Damage Index Items and Smoking (ever/never)

\begin{tabular}{|c|c|c|c|c|c|}
\hline \multicolumn{2}{|c|}{ ALL } & \multicolumn{2}{|c|}{ Caucasian } & \multicolumn{2}{|c|}{ African American } \\
\hline $\begin{array}{l}\text { Damage } \\
\text { Item }\end{array}$ & $\begin{array}{c}\text { OR } \\
(95 \% \\
\text { Cl) }\end{array}$ & p-value & $\begin{array}{c}\text { OR } \\
(95 \% \\
\mathrm{Cl})\end{array}$ & p-value & $\begin{array}{c}\text { OR } \\
(95 \% \\
\mathrm{Cl})\end{array}$ \\
\hline \multicolumn{6}{|l|}{ p-value } \\
\hline $\begin{array}{l}\text { Total } \\
\text { damage }\end{array}$ & $\begin{array}{c}1.73 \\
(1.44 \\
2.07)\end{array}$ & $<0.0001$ & $\begin{array}{c}1.55 \\
(1.22 \\
1.97)\end{array}$ & 0.0003 & $\begin{array}{r}1.96 \\
(1.44 \\
2.66)\end{array}$ \\
\hline$<0.0001$ & & & & & \\
\hline Cataract & $\begin{array}{r}1.50 \\
(1.17 \\
1.92)\end{array}$ & 0.00122 & $\begin{array}{r}0.96 \\
(0.68, \\
1.36)\end{array}$ & 0.8176 & $\begin{array}{l}2.26 \\
(1.56 \\
3.28)\end{array}$ \\
\hline$<0.0001$ & & & & & \\
\hline $\begin{array}{l}\text { Scarring } \\
\text { alopecia }\end{array}$ & $\begin{array}{c}2.08 \\
(1.42, \\
3.06)\end{array}$ & 0.00019 & $\begin{array}{c}1.63 \\
(0.60 \\
4.45)\end{array}$ & 0.3320 & $\begin{array}{r}2.24 \\
(1.46 \\
3.47)\end{array}$ \\
\hline 0.0003 & & & & & \\
\hline $\begin{array}{l}\text { Extensive } \\
\text { scarring or } \\
\text { panniculum } \\
\text { other than } \\
\text { scalp }\end{array}$ & $\begin{array}{c}3.37 \\
(1.95 \\
5.85)\end{array}$ & 0.0001 & $\begin{array}{c}2.57 \\
(1.01 \\
7.03)\end{array}$ & 0.0522 & $\begin{array}{r}3.68 \\
(1.90 \\
7.47)\end{array}$ \\
\hline
\end{tabular}

\begin{tabular}{|c|c|c|c|c|c|}
\hline \multicolumn{6}{|l|}{0.00017} \\
\hline $\begin{array}{l}\text { Skin } \\
\text { ulceration }\end{array}$ & $\begin{array}{l}2.76 \\
(1.37 \\
5.57)\end{array}$ & 0.00465 & $\begin{array}{l}2.03 \\
(0.8 \\
5.36)\end{array}$ & 0.1370 & $\begin{array}{r}4.45 \\
(1.48, \\
16.32)\end{array}$ \\
\hline \multicolumn{6}{|l|}{0.0121} \\
\hline Pulmonary & & & & hypertension & 1.53 \\
\hline & 0.00367 & $\begin{array}{c}1.11 \\
(0.70,1.75)\end{array}$ & 0.6430 & $\begin{array}{c}1.79 \\
(1.21,2.63)\end{array}$ & $\begin{array}{l}(1.15, \\
2.03)\end{array}$ \\
\hline \multicolumn{6}{|l|}{0.00334} \\
\hline $\begin{array}{l}\text { Infarction } \\
\text { or } \\
\text { resection of } \\
\text { bowels } \\
\quad 0.1461\end{array}$ & $\begin{array}{c}1.42 \\
(1.12 \\
1.79)\end{array}$ & 0.0032 & $\begin{array}{c}1.37 \\
(1.03 \\
1.84)\end{array}$ & 0.0338 & $\begin{array}{l}1.36 \\
(0.90, \\
2.04)\end{array}$ \\
\hline Pancreatitis & $\begin{array}{c}3.57 \\
(1.07 \\
11.88)\end{array}$ & 0.0382 & $\begin{array}{l}2.44 \\
(0.68 \\
8.67)\end{array}$ & 0.1694 & $\mathrm{~N} / \mathrm{C}$ \\
\hline \multicolumn{6}{|l|}{$\mathrm{N} / \mathrm{C}$} \\
\hline $\begin{array}{l}\text { Muscle } \\
\text { atrophy }\end{array}$ & $\begin{array}{r}1.80 \\
(1.11 \\
2.94)\end{array}$ & 0.0183 & $\begin{array}{c}2.62 \\
(1.26 \\
5.44)\end{array}$ & 0.0099 & $\begin{array}{l}1.09 \\
(0.54 \\
2.19)\end{array}$ \\
\hline \multicolumn{6}{|l|}{0.8210} \\
\hline $\begin{array}{l}\text { Coronary } \\
\text { artery } \\
\text { disease } \\
0.0226\end{array}$ & $\begin{array}{c}2.26 \\
(1.50 \\
3.41)\end{array}$ & 0.0001 & $\begin{array}{l}2.33 \\
(1.39 \\
3.91)\end{array}$ & 0.0013 & $\begin{array}{l}2.35 \\
(1.13, \\
4.89)\end{array}$ \\
\hline $\begin{array}{l}\text { Myocardial } \\
\text { Infarction }\end{array}$ & $\begin{array}{l}2.30 \\
(1.29 \\
3.32)\end{array}$ & $<0.0001$ & $\begin{array}{l}2.17 \\
(1.30 \\
3.62)\end{array}$ & 0.0032 & $\begin{array}{l}2.22 \\
(1.27, \\
3.87)\end{array}$ \\
\hline \multicolumn{6}{|l|}{0.0051} \\
\hline & & & & Cardiomyopathy & $\begin{array}{l}1.94 \\
(1.29 \\
2.93)\end{array}$ \\
\hline \multirow[t]{2}{*}{0.0016} & $\begin{array}{l}1.64 \\
(0.81 \\
3.30)\end{array}$ & 0.1687 & $\begin{array}{r}2.18 \\
(1.27 \\
3.74)\end{array}$ & 0.0045 & \\
\hline & & & & Claudication & $\begin{array}{l}4.53 \\
(2.17 \\
9.48)\end{array}$ \\
\hline \multirow[t]{2}{*}{$<0.0001$} & $\begin{array}{l}3.86 \\
(1.47, \\
1.10)\end{array}$ & 0.0060 & $\begin{array}{c}4.91 \\
(1.55 \\
15.52)\end{array}$ & 0.0068 & \\
\hline & & & & $\begin{array}{l}\text { Cerebrovascular } \\
\text { accident }\end{array}$ & $\begin{array}{l}1.41 \\
(1.08 \\
1.84)\end{array}$ \\
\hline 0.0127 & $\begin{array}{l}1.16 \\
(0.80 \\
1.69)\end{array}$ & 0.4279 & $\begin{array}{r}1.69 \\
(1.13 \\
2.54)\end{array}$ & 0.0115 & \\
\hline Diabetes & $\begin{array}{c}1.72 \\
(1.29 \\
2.28)\end{array}$ & 0.0002 & $\begin{array}{l}1.33 \\
(0.88 \\
2.03)\end{array}$ & 0.1764 & $\begin{array}{c}2.08 \\
(1.39, \\
3.12)\end{array}$ \\
\hline 0.0004 & & & & & \\
\hline
\end{tabular}

N/C: data are not sufficient for calculating odds ratio

Conclusion: Smoking is a modifiable factor for organ damage in SLE. It is already known that it interferes with the efficacy of hydroxychloroquine. Now we are able to prove that smokers have more cutaneous damage (scarring) even after stratification for ethnicity. As expected, smokers had more cardiovascular damage. New findings include associations with gastrointestinal damage, cataracts, pulmonary hypertension, pancreatitis and diabetes.

Disclosure of Interests: Romy Kallas: None declared, Jessica Li: None declared, Michelle A Petri Shareholder of: Pfizer, Merck, Grant/research support from: AstraZeneca, Exagen, Consultant for: Eli Lilly, GSK, Merck EMD Serono, Janssen, Amgen, Novartis, Quintiles, Exagen, Inova Diagnostics, AstraZeneca, Blackrock, Glenmark, UCB, and the Annenberg Center for Health Sciences

DOI: 10.1136/annrheumdis-2019-eular.5837 